\title{
The role of naldemedine in the treatment of patients with opioid-induced constipation
}

\begin{abstract}
Opioid-induced bowel dysfunction (OIBD) and opioid-induced constipation (OIC) significantly decrease patients' quality of life (QoL), lead to complications and opioid non-compliance resulting in pain exacerbation. Traditional laxatives are first-line preventive and therapeutic measures, although they display limited efficacy and several adverse effects (AE). Non-pharmacology measures, prokinetics, opioid switch, all have little evidence and do not target OIBD and OIC pathophysiology both associated with activation of predominantly $\mu$-opioid receptors mostly peripherally in the gastrointestinal (GI) tract. A combination of prolonged-release (PR) oxycodone with PR naloxone in one tablet with a ratio of 2:1 is available, although limitations include maximal daily dose of $160 \mathrm{mg} / 80 \mathrm{mg}$, respectively, and normal liver function.

Peripherally acting $\mu$-opioid receptor antagonists (PAMORA) block opioid receptors in the Gl tract without compromising analgesia as they do not cross the blood-brain barrier. Currently three drugs are available: methylnaltrexone, naloxegol and naldemedine.

Naldemedine has proven efficacy superior to placebo in the treatment of OIC in both cancer and non-cancer patients while improving patient-reported constipation symptoms and patients' QoL. It is well tolerated with mostly mild to moderate intensity Gl adverse effects such as abdominal, pain, nausea, and diarrhea, without compromising analgesia. Naldemedine dosing is convenient as it is administered once daily by an oral route. Moreover, naldemedine may be safely used in patients with renal failure and mild to moderate hepatic impairment. Effective prevention and treatment of OIC is of paramount importance in patients receiving long-term opioid therapy.
\end{abstract}

Palliat Med Pract 2019; 13, 3: 113-128

Key words: adverse effects, naldemedine, opioid-induced bowel dysfunction; opioid-induced constipation; PAMORA

\section{Introduction}

\section{Opioid-induced constipation}

Opioids are invaluable drugs for the management of chronic severe pain but may also induce numerous adverse effects (AE). Tolerance develops for some of opioid centrally-mediated $\mathrm{AE}$, such as sedation, but often there is little or no tolerance development to peripheral opioid AE including those affecting gastrointestinal (GI) tract function, opioid-induced bowel dysfunction (OIBD). The most common and usually most burdensome for patients symptoms of OIBD

Address for correspondence:

Wojciech Leppert

Laboratory of Quality of Life Research

Poznan University of Medical Sciences

Osiedle Rusa 55

61-245 Poznan, Poland

phone and fax: + 48618738303

e-mail: wojciechleppert@wp.pl 
are called opioid-induced constipation (OIC), which is defined as decreasing number of bowel movements but more importantly subjective symptoms experienced by patients associated with initiating, changing or increasing opioid use. OIBD reflexes the impact of opioids on the whole Gl tract, which comprises such symptoms as dry mouth, gastro-esophageal reflux-related symptoms (heartburn), nausea, vomiting, chronic abdominal pain, bloating, constipation-related symptoms: straining, hard stools, painful, infrequent and incomplete bowel movements, and diarrhea-related symptoms: urgency, loose bowel movements and frequent bowel movements. OIBD and OIC may significantly decrease patients' quality of life (QoL) and lead to non-compliance with opioid regimen resulting in undertreatment of chronic pain. Patients should be closely monitored by the staff to prevent or decrease the intensity of OIBD and OIC. OIBD and OIC are frequent phenomenon with the incidence of approximately $40-80 \%$ patients receiving chronic opioid therapy in both cancer and non-cancer patients [1].

OIBD is associated mainly with the peripheral opioid effect on $\mu$-opioid receptors in the gut wall with probably less central effects. The $\mu$-opioid receptors are located in neurons of myenteric and submucosal plexus. The myenteric plexus is mainly responsible for the control of motor activity of the gut. The submucosal plexus controls the secretion and absorption; $\mu$-opioid receptors are activated in the wall of the stomach, small and large intestine by endogenous and exogenous opioids. Activation of $\mu$-opioid receptors decreases peristaltic contractions and increases GI muscle activity and elevates resting muscle tone, spasm and non-propulsive motility patterns. These mechanisms induce delayed gastric emptying and slow intestinal transit. Additionally opioids increase anal sphincter tone and impair rectal relaxation with distention Opioids increase ileocecal and anal sphincter tones and impair defecation reflex through reduced sensitivity to distension and increased internal anal sphincter tone which may disturb pelvic floor coordination during defecation. The anal sphincter dysfunction is an important factor in the sensation of anal blockage. All these abnormalities result in an increased segmental contraction along with decreased propulsive forward peristalsis. Clinically, these changes manifest as constipation, gut spasm and abdominal cramps. A consequence of this peristaltic disruption is stasis of luminal contents, which leads to an increased passive absorption of fluids, dryer and harder stools [2].

Clinical assessment of patients with symptoms of OIBD and OIC is necessary for the application of effec- tive treatment. It is composed of a detailed history that should include Gl symptoms, other physical problems, psychological and spiritual issues. Physical examination includes palpation of abdomen, auscultation of peristalsis and rectal examination. Acute abdominal diseases should be excluded such as bowel obstruction or gut perforation. Investigations comprise plain radiography, transit time studies, manometry, and anorectal dysfunction tests. However, apart from plain radiography, these investigations are rarely used in palliative care patients [3].

The most commonly use subjective scale is the Bowel Function Index (BFI). The BFI is a three-item questionnaire that assesses the severity of ease of defecation, felling of incomplete bowel evacuation, and patients' personal judgment of constipation using a 0 to 100 numerical rating $(0-$ no symptom, 100 - the most severe symptom). Patients rate these variables of the preceding 7 days. The BFI score is calculated as the mean of the three component scores. Reference results of non-constipated chronic pain patients BFI scores lie within the range of $0-28.8$. The BFI was validated in several clinical studies conducted in cancer and non-cancer patients. Changes in $\mathrm{BFI}$ scores equal or above 12 are likely to be clinically meaningful and those lower than 7.5 unlikely to be clinically relevant, with changes between 7.5 and 12 not clear that require further evaluation [4].

The Patient Assessment of Constipation includes the symptom questionnaire (PAC-SYM) and the quality of life questionnaire (PAC-QOL). The PAC-SYM contains 12 items assigned to three subscales: stool symptoms, rectal symptoms, and abdominal symptoms. PAC-SYM also contains a question about the frequency of bowel movements during the last 7 days [40-9]. The PACQOL is composed of 28 items grouped into 4 subscales related to dissatisfaction (5 items), physical discomfort (4 items), psychosocial discomfort (8 items), and worries and concerns (11 items). Validation studies have confirmed that PAC-QOL is internally consistent, reproducible, valid, and responsive to improvements over time [5]. The objective assessment comprises the Bristol Stool Chart - a simple and easy to use method evaluating stool frequency and stool consistency. However, only a moderate correlation between stool form and whole-gut or colonic transit time can be demonstrated [6].

\section{Management of opioid-induced constipation}

\section{Non-pharmacological measures}

Non-pharmacological measures may be considered throughout a whole period of care and play 
an important role in the prevention of OIBD and OIC development. This may include ensuring privacy and comfort to allow a patient to defaecate normally, appropriate positioning (to assist gravity, a small footstool may help patient exert pressure more easily), increased fluid intake, encourage activity and increased mobility within patient limits (even bed to chair), anticipatory management of constipation when opioids prescribed, advise against home remedies or "over the counter" or online products which may impact treatments. An important role of education of patients' and careers should be emphasized [1].

\section{Prokinetics}

Itopride is a peripheral antidopaminergic agent that also increases acetylocholine level due to the inhibition of acetylocholinesterase and the prokinetic effect observed in the whole GI tract. It does not activate $5-\mathrm{HT}_{4}$ nor $5-\mathrm{HT}_{3}$ receptors. Itopride displays little risk of significant pharmacokinetic interactions with other drugs. It does not permeate the blood-brain barrier and poses no risk of extra-pyramidal effects [7]. The recommended dose is usually $50 \mathrm{mg}$ three times daily.

Prucalopride is a selective $5 \mathrm{HT}_{4}$ receptor agonist which stimulates propulsive gut motility. Prucalopride accelerates the whole gut, gastric, small bowel and colonic transit in patients with chronic constipation [8]. The recommended dose is usually $2 \mathrm{mg}$ once daily. Prucalopride was introduced for the treatment of chronic constipation, predominantly in women [9]. Itopride and prucalopride do not display cardiac toxicity.

Lubiprostone activates type- 2 chloride channels and increases intestinal fluid secretion, thereby facilitating intestinal transit and increasing the passage of stool [10]. Lubiprostone is registered by the FDA for the management of OIBD in patients with chronic non-malignant pain. The most common AE comprised nausea ( $11 \%$ of treated patients), diarrhea $(8 \%)$, and abdominal pain (4\%). However, in contrast to morphine lubiprostone may be ineffective in patients treated with methadone [11].

Linaclotide is an agent that regulates chloride secretion on the intestinal epithelial cells through activation of guanylate cyclase $C$. Linaclotide stimulates the Gl secretion and transit and also modulates visceral sensitivity. It increases stool frequen$c y$, stool weight and improves the ease of stool passage. The main AE of linaclotide is diarrhea [12]. Linaclotide may be potentially considered for the management of OIBD although it is used in patients with chronic constipation and irritable bowel syndrome [13].

\section{Laxatives}

Numerous laxatives are prescribed for the prevention and treatment of OIBD, particularly for constipation-related symptoms. The most commonly used group of laxatives for OIBD comprise osmotic agents and stimulants. Nonetheless, they possess limited efficacy not proven in RCT and display their own $A E$ [14]. Moreover, treatment with laxatives should be limited to a short period of time. Patients with chronic non-malignant and cancer-related pain usually have to take laxatives for the long-term [15].

\section{Rectal measures}

Suppositories and enemas are a preferred first-line therapy when digital rectal examination identifies a full rectum or fecal impaction. Suppositories and enemas may be perceived as a more invasive option by patients. Enemas (such as hyperosmotic saline) and suppositories increase water content and stimulate peristalsis to aid in expulsion, and both work more quickly than oral laxatives. In general, enemas are used only if oral treatment fails after several days and in order to prevent fecal impaction [2].

\section{Change of opioid route of administration and opioid rotation (switch)}

A change from oral to parenteral or to transdermal route of opioid administration may be considered. However, all opioids display OIBD including transdermal formulations. A switch from hydrophilic opioids (morphine, oxycodone, hydromorphone) to lipophylic drugs (fentanyl, buprenorphine, methadone) due to less peripheral effects of the latter may be considered, although the evidence is limited [1].

\section{Combination of opioid receptors agonist with opioid receptors antagonists}

Newer strategies for the management of OIBD include administration of a combination of prolonged-release (PR) oxycodone with PR naloxone (OXN) in one tablet. OXN renders analgesia with limited negative effect of oxycodone on bowel function. Naloxone display very low systemic availability (approximately $2 \%$ ) after oral administration of PR formulation. However, limitations of OXN use include maximal daily dose of $160 \mathrm{mg} / 80 \mathrm{mg}$ of oxycodone and naloxone, respective$l y$, and a requirement of a normal liver function and portal circulation necessary to avoid significant systemic availability and the risk of opioid withdrawal [16].

\section{Peripherally acting $\mu$-opioid receptor antagonists}

Treatment with opioid receptor antagonists that act purely peripherally may be used when traditional oral laxatives fail. 


\section{Methylnaltrexone}

MNTX is a quaternary methyl derivative of naltrexone, preferential, non-selective, peripheral $\mu$-opioid receptor antagonist. The addition of the methyl group to the nitrogen ring increases polarity and reduces lipophilicity of MNTX. As a consequence, MNTX does not cross the blood-brain barrier and displays limited absorption from the gut. When administered systemically (orally, subcutaneously, intravenously), MNTX does not reverse opioid analgesia, which is the case for MNTX intrathecal administration [17].

MNTX is effective in palliative care patients with OIC when conventional laxatives have failed. A systematic review and meta-analysis of 14 controlled studies including 4101 patients demonstrated superiority of mu-opioid receptor antagonists over placebo in the treatment of OIC. MNTX, naloxone and alvimopan (used only for up to 48 hours in the treatment of postoperative ileus due to the risk of cardiotoxicity) all were superior to placebo. AE (diarrhea and abdominal pain) were more common during these drugs administration compared to placebo. Reversal of analgesia was not observed during active therapy. Lubiprostone was beneficial in two controlled studies and prucalopride showed a tendency of a better response in one study but the meta-analysis could not be conducted [18].

MNTX dosing (a single dose administered by subcutaneous route equals 8-12 mg depending on the body weight) is similar in younger and older patients. No dose adjustment is required in patients with mild to moderate renal impairment. However, in patients with renal failure (creatinine clearance $<30 \mathrm{~mL} / \mathrm{min}$ ) the MNTX dose should be reduced by one-half. No dose adjustment is required for patients with mild to moderate hepatic impairment. MNTX is not recommended for patients with end-stage renal impairment requiring dialysis and for patients with severe liver impairment [17].

It is recommended that MNTX should be used with caution in patients with present or suspected lesions of the Gl tract. Treatment with MNTX should be stopped if severe abdominal symptoms appear or intensify. If severe or persistent diarrhea occurs during the treatment, patients are advised to discontinue the MNTX therapy. Clinical studies demonstrated the effectiveness of MNTX in the treatment of OIC not responding to traditional laxatives in patients with advanced diseases, non-malignant pain [17].

\section{Naloxegol}

Naloxegol is a polymer conjugate of the opioid antagonist naloxone administered once daily by the oral route. The polyethylene glycol moiety limits naloxegol capacity to cross the blood-brain barrier. In a phase 2 , randomized, double-blind, placebo-controlled, dose-escalation study, the efficacy and safety of three dose levels of oral naloxegol in the treatment of OIC in patients with non-malignant or cancer-related pain was evaluated. The dose of $25 \mathrm{mg}$ naloxegol was chosen to be further explored in phase 3 studies [19].

Naloxegol is metabolised through CYP3A4 to six metabolites, with the majority of the dose $(68 \%)$ excreted with faeces and less (16\%) with urine. The dose of naloxegol equals $25 \mathrm{mg}$ administered orally once daily on a fasting condition. Mild or moderate hepatic impairment has no impact on naloxegol dosing; naloxegol was not studied and is not recommended in patients with hepatic failure. Dose reduction $(12.5 \mathrm{mg}$ once daily) and caution is recommended in patients with moderate-to-severe renal impairment. Efficacy measured as bowel movement (BM) was noted in $42-49 \%$ of patients not responsive to laxatives. Safety of naloxegol were confirmed in studies conducted in patients with OIC and non-malignant pain.

Two phase-3, double-blind clinical studies (KODIAC-04 and KODIAC-05) have been completed and demonstrated significant increase in spontaneous bowel movements (SBM) compared to placebo for the $25-\mathrm{mg}$ naloxegol dose (in one study also for the $12.5-\mathrm{mg}$ dose), with arthralgia as the only $\mathrm{AE}$ more common than in the placebo group [20]. In both studies (study 04, 652 participants; study 05, 700 participants), outpatients with non-cancer pain and OIC were randomly assigned to receive a daily dose of 12.5 or $25 \mathrm{mg}$ of naloxegol or placebo. The primary end point was the 12-week response rate ( $\geq 3$ SBM per week and an increase from baseline of $\geq 1$ SBM for $\geq 9$ of 12 weeks and for $\geq 3$ of the final 4 weeks) in the intention-to-treat population. The key secondary end points were the response rate in the subpopulation of patients with an inadequate response to laxatives before enrollment, time to first post-dose SBM, and mean number of days per week with one or more SBM.

Response rates were significantly higher with $25 \mathrm{mg}$ of naloxegol than with placebo (intention-to-treat population: study $04,44.4 \%$ vs. $29.4 \%, \mathrm{P}=0.001$; study $05,39.7 \%$ vs. $29.3 \%, P=0.02$; patients with an inadequate response to laxatives: study $04,48.7 \%$ vs. $28.8 \%, \mathrm{P}=0.002$; study $05,46.8 \%$ vs. $31.4 \%$, $\mathrm{P}=0.01$ ); in study 04 , response rates were also higher in the group treated with $12.5 \mathrm{mg}$ of naloxegol (intention-to-treat population, $40.8 \%$ vs. $29.4 \%, \mathrm{P}=0.02$; patients with an inadequate response to laxatives, $42.6 \%$ vs. $28.8 \%, P=0.03$ ). A shorter time to the first post-dose SBM and a higher mean number of days per week with one or more SBM were observed with 
$25 \mathrm{mg}$ of naloxegol versus placebo in both studies $(P<0.001)$ and with $12.5 \mathrm{mg}$ of naloxegol in study $04(P<0.001)$. Pain scores and daily opioid dose were similar among the three groups. AE (primarily gastrointestinal) occurred most frequently in the groups treated with $25 \mathrm{mg}$ of naloxegol. Treatment with naloxegol, as compared with placebo, resulted in a significantly higher rate of treatment response, without reducing opioid-mediated analgesia [20].

\section{Naldemedine}

Naldemedine is an antagonist of the mu, delta, and kappa opioid receptors. It acts as PAMORA in the $\mathrm{GI}$ tract and decrease $\mathrm{OIC}$ without reversing analgesia which is mediated by the opioid action in the CNS. Naldemedine is a derivative of naltrexone with a side chain added, which increases the molecular weight and the polar surface area and thus reducing its ability to cross the blood-brain barrier (BBB) [21].

\section{Pharmcodynamics}

In an experimental study the binding affinity and antagonist activity of naldemedine against recombinant human $\mu-, \delta$-, and $\kappa$-opioid receptors were assayed in vitro [22]. Pharmacologic effects of naldemedine were investigated using animal models of morphine-induced inhibition of small and large intestinal transit, castor oil-induced diarrhea, antinociception, and morphine withdrawal. Naldemedine showed potent binding affinity and antagonist activities for recombinant human $\mu-, \delta$-, and $\kappa$-opioid receptors. Naldemedine significantly reduced opioid-induced inhibition of small intestinal transit $(0.03-10 \mathrm{mg} / \mathrm{kg} ; \mathrm{P}<0.05)$ and large intestinal transit $(0.3-10 \mu \mathrm{mol} / \mathrm{L} ; \mathrm{P}<0.05)$. Naldemedine $(0.03-1 \mathrm{mg} / \mathrm{kg}$ ) pretreatment significantly reversed the inhibition of castor oil-induced diarrhea by subcutaneous morphine $(P<0.01)$. Naldemedine (1-30 $\mathrm{mg} / \mathrm{kg}$ ) pretreatment ( 1 or 2 hours) did not alter the analgesic effects of morphine in a tail flick test in rats, although significant delayed reduction of the analgesic effect of morphine was seen with higher doses of naldemedine $(10-30 \mathrm{mg} / \mathrm{kg})$. Some centrally mediated and peripherally mediated withdrawal signs in morphine-dependent rats were seen with naldemedine doses $\geq 3$ and $\geq 0.3 \mathrm{mg} / \mathrm{kg}$, respectively. Naldemedine displayed potent binding affinity to, and antagonistic activity against, $\mu-, \delta-$, and $\kappa$-opioid receptors. Naldemedine decreased OIC in vivo without compromising opioid analgesia [22].

The CNS penetration of naldemedine is negligible when administered at the recommended dose. It is a substrate of the P-glycoprotein (P-gp) efflux transporter, which may also decrease naldemedine penetration into the CNS. Following a single oral ad- ministration of $\left[{ }^{14} \mathrm{C}\right]$-naldemedine tosylate to rats and ferrets little radioactivity was detected in the region protected by the BBB. The contribution of P-gp to the brain distribution of naldemedine was assessed using multidrug resistance $1 \mathrm{a} / \mathrm{b}$ (mdr1a/b) knockout mice [23]. While the brain-to-plasma concentration ratio (brain $\mathrm{Kp}$ ) of naldemedine in the $\mathrm{mdr} 1 \mathrm{a} / \mathrm{b}$ knockout mice was 4-fold of that in the wild-type mice, the brain $\mathrm{Kp}$ in the $\mathrm{mdr} 1 \mathrm{a} / \mathrm{b}$ knockout mice was quite low (brain $\mathrm{Kp}<0.1$ ) suggesting that the low brain distribution of naldemedine was due to the limited ability to cross the BBB rather than efflux by P-gp and therefore brain distribution of naldemedine would not be affected by concurrent administration of P-gp inhibitors or functional disorder of P-gp [23]. As a consequence naldemedine decrease constipating effects of opioids without compromising CNS-mediated opioid analgesia.

\section{Pharmacokinetics}

Naldemedine is absorbed from Gl tract achieving peak plasma concentration of approximately 45 minutes in the fasted state. The absolute bioavailability of naldemedine is in the range of $20 \%$ to $56 \%$ without clinically significant food effect. The peak plasma concentration was reduced by $35 \%$ and time to achieve peak plasma concentration was delayed from $45 \mathrm{mi}$ nutes in the fasted state to 2.5 hours in the fed state, whereas no significant difference was observed in the area under the plasma concentration-time curve by food intake. Thus, naldemedine can be taken with or without food. Naldemedine is highly bound to predominantly albumin and to a lesser extent to alfa1-acid-glycoprotein and gamma-globulin, with a mean protein binding ration of $93.2 \%$. The volume of distribution equals approximately 155 litres [24].

Naldemedine is metabolized by CYP3A to nor-naldemedine, with a minor contribution from UGT1A3 to form naldemedine-3-glucuronide (N-3-G). The main metabolite in is nor-naldemedine, with a relative exposure compared to naldemedine of approximately 9 to $13 \%$. N-3-G is a minor metabolite, with a relative exposure to naldemedine of less than $3 \%$. Naldemedine also undergoes cleavage in the Gl tract to form benzamidine and naldemedine carboxylic acid.

Naldemedine does not inhibit or induce most CYP enzymes (including CYP1A2, CYP2A6, CYP2B6, CYP2C8, CYP2C9, CYP2C19, CYP2D6, CYP2E1, CYP3A, or CYP4A11 isozymes) and does not inhibitor major transporters, thus it probably does not affect the pharmacokinetics of concurrently administered drugs.

The elimination half-life of naldemedine is approximately 11 hours, and the total clearance (CL/F) of naldemedine is $8.4 \mathrm{~L} / \mathrm{h}$. Naldemedine is excreted 
in urine and faeces with approximately $20 \%$ of the naldemedine dose excreted unchanged in urine [4]. The peak plasma concentration and area under the plasma concentration-time curve increased in an almost dose-proportional manner within the dose range of 0.1 to $100 \mathrm{mg}$. A slight accumulation (1 to 1.3 -fold) for peak plasma concentration and area under the plasma concentration-time curve was observed after once daily multiple dose administration in the fasted state for 10 days.

Mild, moderate and severe renal impairment and dialysis does not affect significantly naldemedine pharmacokinetics. A mild and moderate hepatic impairment does not significantly affect naldemedine pharmacokinetics. However, patients with severe hepatic impairment were not studied, therefore naldemedine is not recommended in this patient group [25].

\section{Adverse effects}

The most commonly reported adverse reactions in patients with chronic non-cancer pain and OIC were abdominal pain $(7.8 \%)$, diarrhea $(5.9 \%)$, nausea (3.6\%), and vomiting (1.1\%). The majority of these $\mathrm{Gl}$ adverse reactions were of mild to moderate severity and resolved without discontinuation of naldemedine treatment. One serious case of abdominal pain and one serious case of nausea were reported in patients with chronic non-cancer pain and OIC. The most commonly reported adverse reactions in patients with cancer and OIC were diarrhea (24.5\%) and abdominal pain (3.9\%). The majority of these gastrointestinal adverse reactions were of mild to moderate severity and resolved with treatment. Two serious cases of diarrhea were reported in patients with cancer and OIC [26].

Naldemedine is indicated for the treatment of opioid-induced constipation (OIC) in adult patients who have previously been treated with a laxative. Contraindications comprise hypersensitivity to the active substance or to any of the excipients of tablet. Patients with known or suspected Gl obstruction or perforation or patients at increased risk of recurrent obstruction, due to the potential for Gl perforation [26]. Patients should be monitored for the development of severe, persistent or worsening abdominal pain. If obstruction or perforation are suspected, naldemedine must be discontinued.

Caution should be exercised with regards to opioid withdrawal. Patients should be advised to discontinue naldemedine and to contact their physician if opioid withdrawal occurs. Cases of possible opioid withdrawal syndrome have been reported in the naldemedine clinical program. Patients having disruptions to the blood-brain barrier such as primary brain primary malignancies, brain metastases or other inflammatory conditions, active multiple sclerosis and advanced Alzheimer's disease may be at increased risk of opioid withdrawal or reduced analgesia. The overall benefit-risk of naldemedine should be considered in these patients with close monitoring for symptoms of opioid withdrawal.

The use of naldemedine during pregnancy may precipitate opioid withdrawal in a neonate due to the immature fetal blood brain barrier. Naldemedine should not be used during pregnancy unless the clinical condition of the woman requires treatment with naldemedine. It is unknown whether naldemedine and its metabolites are excreted in human milk, although naldemedine is excreted in milk in rats. At therapeutic doses, most opioids are excreted into breast milk in minimal amounts. There is a theoretical possibility that naldemedine provokes opioid withdrawal in a breast-fed neonate whose mother is taking an opioid receptor agonist, thus naldemedine should not be used during breast-feeding [6].

\section{Overview of naldemedine clinical studies}

Based on the results of phase II studies $0.2 \mathrm{mg}$ naldemedine was selected as an optimal dose of phase III clinical studies [27, 28].

\section{Studies in non-cancer patients}

Two phase III randomized, double-blind, placebo-controlled clinical trials (COMPOSE-I, NCT 01965158; COMPOSE-II, NCT 01993940) were conducted to assess the efficacy and safety of a daily dose of $0.2 \mathrm{mg}$ naldemedine [29]. Adults on a stable opioid regimen who discontinued prior laxatives were randomized based on their baseline opioid consumption. Patients received active treatment for 12 weeks, with subsequent monitoring for an additional 4 weeks. Based on an intention-to-treat population, COMPOSE-I (273 patients naldemedine; 272 placebo) and COMPOSE-II (276 patients naldemedine; 274 placebo) tested a durable response endpoint defined as $\geq 3$ SBM per week and an increase of $\geq 1$ SBM per week from baseline for at least nine of the 12 treatment weeks, inclusive of at least three of the last 4 weeks. In both studies, there were significantly more responders in the naldemedine group compared to placebo (COMPOSE-I: 47.6 vs. $34.6 \%, \mathrm{P}=0.002$, COMPOSE-II: 52.5 vs. $33.6 \%, \mathrm{P}<0.0001$ ). There were also significant increases in the mean frequency of SBM per week from baseline to the last 2 weeks of treatment in favor of naldemedine (COMPOSE-I: least square mean increase $3.42 \pm 0.193$ [standard error] vs. $2.12 \pm 0.192, \mathrm{P}<0.0001$, and COMPOSE-II: $3.56 \pm 0.174$ vs. $2.16 \pm 0.174, \mathrm{P}<0.001)$. In both 
studies, there were similar numbers of $A E$ in both groups (COMPOSE-I: 49 vs. $45 \%$ and COMPOSE-II: 50 vs. $48 \%$ ). A higher proportion of treatment-related adverse effects (TRAE) were noted in the naldemedine groups relative to placebo in both studies (COMPOSE-I: 22 vs. $17 \%$ and COMPOSE-II: 20 vs. $11 \%$ ). The most frequently reported AE were diarrhea (COMPOSE-I: 7 vs. $3 \%$ and COMPOSE-II: 9 vs. $2 \%$ ) and abdominal pain (COMPOSE-I: 6 vs. $2 \%$ and COMPOSE-II: 5 vs. $1 \%$ ). Two major cardiovascular AE were documented, one in a treatment patient in COMPOSE-I and one in a placebo patient in COMPOSE-II. One patient receiving naldemedine in the COMPOSE-II study died from an opioid overdose leading to cardiopulmonary arrest. Three patients in COMPOSE-I (two naldemedine and one placebo) experienced symptoms of opioid withdrawal [29].

The final Phase III trial (COMPOSE-III, NCT 01965652) was a 52-week multicenter randomized, double-blind, placebo-controlled study evaluating the long-term efficacy and safety of once daily $0.2 \mathrm{mg}$ naldemedine [30]. The study population consisted of adults with OIC on stable doses of opioids, who may or may not have been on other laxatives. The primary outcome was safety and tolerability of naldemedine. Secondary endpoints assessed naldemedine efficacy. A total of 620 naldemedine and 620 placebo patients were included in the safety analysis. There were similar treatment-emergent adverse effects (TEAE) in each group (68.4 vs. $72.1 \%$, respectively). TEAE that occurred in $>5 \%$ of patients and with a higher frequency in naldemedine than placebo groups included abdominal pain (8.2 vs. $3.1 \%$ ), diarrhea (11.0 vs. $5.3 \%$ ), and vomiting ( 6.0 vs. $3.1 \%$ ). No associations between naldemedine and opioid withdrawal or decreased analgesia were evident. Patients in the treatment group had more frequent bowel movements (BM) per week, and this difference was statistically significant at all measured time points including at 52 weeks [30].

In two open-label, single-arm, Phase III studies, the safety and efficacy of naldemedine was assessed in Japanese patients with OIC receiving regular-use opioids (COMPOSE-6) or PR oxycodone (COMPOSE-7) for chronic noncancer pain [31]. Eligible Japanese adults with $\mathrm{OIC}$ and chronic noncancer pain received once-daily oral naldemedine $0.2 \mathrm{mg}$ for 48 weeks, irrespective of food intake. Primary end points included measures of TEAE, pain intensity, and opioid withdrawal. Secondary efficacy end points were evaluated at treatment week 2. PAC-SYM and PAC-QOL scores were evaluated in both 48-week studies.

Of patients enrolled in COMPOSE- $6(\mathrm{~N}=43)$ and COMPOSE-7 $(\mathrm{N}=10)$, TEAE were reported in $88 \%$ (95\% Cl 74.9-96.1) and 90\% (95\% Cl 55.5-99.7), respectively. The most frequently reported TEAE, nasopharyngitis and diarrhea, were mostly mild or moderate in severity. Assessments of pain intensity and opioid withdrawal remained stable over the 48-week treatment periods of both studies. The proportion of SBM responders at week 2 in COMPOSE- 6 was $81.0 \%$ (95\% Cl 65.9-91.4) and $90.0 \%$ (95\% Cl 55.5-99.7) in COMPOSE-7. Significant and sustained improvements in PAC-SYM and PAC-QOL scores were also observed in both studies (all $p<0.05$ ). AE of naldemedine were mostly mild or moderate in severity, and the results suggest that naldemedine improve bowel function and QOL in Japanese patients with OIC receiving regular-use opioids or PR oxycodone for chronic noncancer pain [31].

In an exploratory analysis [32], the onset of action of naldemedine was evaluated in two phase 3 , randomized, placebo-controlled trials [29]. Proportion of patients experiencing a SBM within 24 hours of treatment initiation, time from initial dose to first SBM and weekly SBM frequency were assessed. Naldemedine was associated with significant increases in the proportion of patients experiencing SBM at 4, 8, 12, and 24 hours after the initial dose compared with placebo (all $P<0.0001$ ). Within 24 hours in both studies, statistically significantly $(P<0.0001)$ more patients treated with naldemedine compared with placebo experienced an SBM (61.2\% vs. $28.3 \%$ and $56.5 \%$ vs. $33.6 \%$, respectively). Median times to first SBM were significantly shorter in the naldemedine group vs. placebo (COMPOSE-1, 16.1 vs. 46.7 hours; COMPOSE-2, 18.3 vs. 45.9 hours; $P<0.0001$ ). Naldemedine was also associated with significant increases in weekly SBM frequency vs. placebo within 1 week $(P<0.001)$. Most common TEAE were Gl-related (abdominal pain, diarrhea, and nausea). TEAE were reported most frequently on day 1 , followed by a decrease from days 2 to 7 . Naldemedine had a timely onset of effect, and $\mathrm{Gl}$ adverse events largely resolved within the first week. These findings should assist clinicians counseling patients with chronic noncancer pain on expectations when initiating naldemedine for OIC [32].

\section{Studies in cancer patients}

A phase III trial consisted of a 2-week, randomized, double-blind, placebo-controlled study (COMPOSE-4) and an open-label, 12-week extension study (COMPOSE-5) [33]. In COMPOSE-4, eligible adults with OIC and cancer were randomly assigned on a 1:1 basis to receive once-daily oral naldemedine $0.2 \mathrm{mg}$ or placebo. The primary end point was the proportion of spontaneous bowel movement (SBM) responders ( $\geq 3 \mathrm{SBM} /$ week and an increase 
of $\geq 1 \mathrm{SBM} /$ week from baseline). The primary end point of COMPOSE- 5 was safety.

In COMPOSE-4, 193 eligible patients were randomly assigned to naldemedine $(n=97)$ or placebo $(n=96)$. The proportion of SBM responders in COMPOSE-4 was significantly greater with naldemedine than with placebo (71.1\% [69 of 97 patients] vs. $34.4 \%$ [33 of 96 patients]; $P<0.0001$ ). A greater change from baseline was observed with naldemedine than with placebo in the frequency of SBMs/week (5.16 vs. 1.54; $P<0.0001)$, SBM with complete bowel evacuation/week (2.76 vs. 0.71; P $<0.0001)$, and SBM without straining/week (3.85 vs. 1.17; $\mathrm{P}=0.0005$ ). In COMPOSE-4, more patients treated with naldemedine than with placebo reported TEAE $(44.3 \%$ [43 of 97 patients] vs. $26.0 \%$ [25 of 96 patients]; $P=0.01)$; in COMPOSE-5, 105 (80.2\%) of 131 of patients reported TEAEs. Diarrhea was the most frequently reported TEAE in COMPOSE-4 (19.6\% [19 of 97 patients] vs. 7.3\% [seven of 96 patients] with naldemedine vs. placebo) and COMPOSE-5 (18.3\% [24 of 131 patients] with naldemedine). Naldemedine was not associated with signs or symptoms of opioid withdrawal and had no notable impact on opioid-mediated analgesia. Once-daily oral naldemedine $0.2 \mathrm{mg}$ effectively treated OIC and was generally well tolerated in cancer patients with OIC [33].

In another report [34] results from secondary end points, including quality of life (QOL) assessments from a phase III, double-blind study (COMPOSE-4) and its open-label extension (COMPOSE-5) [33] was presented [34].

In COMPOSE-4, eligible adults with OIC and cancer were randomly assigned 1:1 to receive once daily oral naldemedine $0.2 \mathrm{mg}(\mathrm{n}=97)$ or placebo $(\mathrm{n}=96)$ for 2 weeks, and those who continued on to COMPOSE-5 received naldemedine for 12 weeks ( $n=131$ ). Secondary assessments in COMPOSE-4 included the proportion of complete SBM (CSBM) responders, SBM or CSBM responders by week, and subjects with $\geq 1$ SBM or CSBM within $24 \mathrm{~h}$ postinitial dose. Changes from baseline in the frequency of SBMs or CSBMs per week were assessed at weeks 1 and 2. Time to the first SBM or CSBM postinitial dose was also evaluated. In both studies, QOL impact was evaluated by PAC-SYM and PAC-QOL questionnaires.

Naldemedine improved bowel function for all secondary efficacy assessments versus placebo (all $P \leq 0.0002$ ). The timely onset of naldemedine activity versus placebo was evidenced by median time to the first SBM (4.7 $\mathrm{h}$ vs. $26.6 \mathrm{~h})$ and CSBM (24.0 h vs. $218.5 \mathrm{~h}$ ) postinitial dose (all $\mathrm{P}<0.0001$ ). In COMPOSE-4, significant differences between groups were observed with the PAC-SYM stool domain ( $P=0.045)$ and PAC-QOL dissatisfaction domain ( $P=0.015)$. In COMPOSE-5, significant improvements from baseline were observed for overall and individual domain scores of PAC-SYM and PAC-QOL. Naldemedine provided effective and timely symptomatic relief from OIC and improved the QOL of subjects with OIC and cancer [34].

In a post-hoc, pooled, subgroup analysis [35] of two randomized studies $[28,33]$ baseline characteristics was evaluated that may influence the efficacy and safety of naldemedine in patients with OIC and cancer [35]. Data for patients who received $0.2 \mathrm{mg}$ naldemedine or placebo were pooled from randomized, placebo-controlled, phase IIb and phase III studies. Proportions of SBM responders and patients with diarrhea were assessed for each treatment group. For the patient subgroups with or without possible BBB disruptions, changes in Numerical Rating Scale (NRS) and Clinical Opioid Withdrawal Scale (COWS) scores were assessed.

A total of 307 patients were included in this analysis (naldemedine: 155; placebo: 152). The pooled proportion of SBM responders was $73.5 \%$ with naldemedine vs. $35.5 \%$ with placebo. There was a significant increase in the proportion of SBM responders with naldemedine vs. placebo $38.0 \%$ (95\% Cl 27.6-48.4\%; $\mathrm{p}<0.0001)$. Greater proportions of SBM responders and patients who experienced diarrhea were observed with naldemedine vs. placebo in all subgroups. Changes from baseline in NRS and COWS scores were similar with naldemedine or placebo in patients with or without brain metastases [35].

Although not powered to detect statistically significant differences in treatment effect among subgroups, this study demonstrated that naldemedine appeared to benefit cancer patients with OIC and cancer, irrespective of baseline characteristics, and did not seem to affect analgesia or withdrawal even in patients with potential BBB disruptions. Baseline characteristics did not appear to affect the incidence of diarrhea in patients who received naldemedine [35].

\section{Summary}

OIBD and OIC are common complication in patients receiving long-term opioid therapy. Any intervention should always be preceded with a meticulous assessment of the OIC taking into account the underlying disease, co-morbidities and a thorough patient's assessment including non-medical problems [36]. A significant progress has been made with targeted therapies for the management of patients with symptoms of OIC and OIBD, namely OXN, MNTX and PAMORA designed for oral route of administration 
such as naloxegol and naldemedine [37-39]. Expert Group of the European Society of Medical Oncology and Polish Association for Palliative Medicine recommend a consideration of PAMORA in the management of OIC $[40,41]$. Current data from clinical studies and systematic reviews indicate that naldemedine is an effective and generally well-tolerated treatment option for OIC in patients with cancer pain or chronic non-cancer pain, with the convenience of once-daily oral dosing $[42,43]$.

\section{References}

1. Brock $C$, Olesen $S$, Olesen $A$, et al. Opioid-Induced Bowel Dysfunction. Drugs. 2012; 72(14): 1847-1865, doi: 10.2165/11634970-000000000-00000.

2. Reimer K, Hopp M, Zenz M, et al. Meeting the challenges of opioid-induced constipation in chronic pain management - a novel approach. Pharmacology. 2009; 83(1): 10-17, doi: 10.1159/000165778, indexed in Pubmed: 18957874.

3. Olesen $A E$, Drewes $A M$. Validated tools for evaluating opioid-induced bowel dysfunction. Adv Ther. 2011; 28(4): 279-294, doi: 10.1007/s12325-011-0006-4, indexed in Pubmed: 21437762.

4. Abramowitz L, Béziaud N, Caussé C, et al. Further validation of the psychometric properties of the Bowel Function Index for evaluating opioid-induced constipation (OIC). J Med Econ. 2013; 16(12): 1434-1441, doi: 10.3111/13696998.2013.851083, indexed in Pubmed: 24102611.

5. Marquis P, De La Loge C, Dubois D, et al. Development and validation of the Patient Assessment of Constipation Quality of Life questionnaire. Scand J Gastroenterol. 2005; 40(5): 540-551, doi: 10.1080/00365520510012208, indexed in Pubmed: 16036506.

6. Lewis SJ, Heaton KW. Stool form scale as a useful guide to intestinal transit time. Scand J Gastroenterol. 1997; 32(9): 920-924, doi: 10.3109/00365529709011203, indexed in Pubmed: 9299672.

7. Chojnacki J. Itopride in the treatment of kinetic disorders of gastrointestinal tract. Gastroenterology Review. 2011; 3: 139-145, doi: 10.5114/pg.2011.23139.

8. Bouras EP, Camilleri M, Burton DD, et al. Prucalopride accelerates gastrointestinal and colonic transit in patients with constipation without a rectal evacuation disorder. Gastroenterology. 2001; 120(2): 354-360, doi: 10.1053/gast.2001.21166, indexed in Pubmed: 11159875.

9. Tack J, van Outryve M, Beyens G, et al. Prucalopride (Resolor) in the treatment of severe chronic constipation in patients dissatisfied with laxatives. Gut. 2009; 58(3): 357-365, doi: 10.1136/gut.2008.162404, indexed in Pubmed: 18987031.

10. Thayalasekeran S, Ali H, Tsai HH. Novel therapies for constipation. World J Gastroenterol. 2013; 19(45): 82478251, doi: 10.3748/wjg.v19.i45.8247, indexed in Pubmed: 24363515.

11. Cuppoletti J, Chakrabarti J, Tewari K, et al. Methadone but not morphine inhibits lubiprostone-stimulated $\mathrm{Cl}$ - currents in T84 intestinal cells and recombinant human $\mathrm{ClC}-2$, but not CFTR Cl- currents. Cell Biochem Biophys. 2013; 66(1): 53-63, doi: 10.1007/s12013-012-9406-6, indexed in Pubmed: 22918821.

12. Müller-Lissner S. Pharmacokinetic and pharmacodynamic considerations for the current chronic constipation treatments. Expert Opin Drug Metab Toxicol. 2013; 9(4):
391-401, doi: 10.1517/17425255.2013.773972, indexed in Pubmed: 23425050.

13. Busby RW, Kessler MM, Bartolini WP, et al. Pharmacologic properties, metabolism, and disposition of linaclotide, a novel therapeutic peptide approved for the treatment of irritable bowel syndrome with constipation and chronic idiopathic constipation. J Pharmacol Exp Ther. 2013; 344(1): 196-206, doi: 10.1124/jpet.112.199430, indexed in Pubmed: 23090647.

14. Larkin PJ, Sykes NP, Centeno C, et al. European Consensus Group on Constipation in Palliative Care. The management of constipation in palliative care: clinical practice recommendations. Palliat Med. 2008; 22(7): 796-807. doi: 10.1177/0269216308096908, indexed in Pubmed: 18838491.

15. Holzer P, Ahmedzai SH, Niederle N, et al. Opioid-induced bowel dysfunction in cancer-related pain: causes, consequences, and a novel approach for its management. J Opioid Manag. 2009; 5(3): 145-151, indexed in Pubmed: 19662924.

16. Meissner W, Schmidt U, Hartmann M, et al. Oral naloxone reverses opioid-associated constipation. Pain. 2000; 84(1): 105-109, doi: 10.1016/s0304-3959(99)00185-2, indexed in Pubmed: 10601678.

17. Thomas J, Karver S, Cooney GA, et al. Methylnaltrexone for opioid-induced constipation in advanced illness. N Engl J Med. 2008; 358(22): 2332-2343, doi: 10.1056/NEJMoa0707377, indexed in Pubmed: 18509120.

18. Ford AC, Brenner DM, Schoenfeld PS. Efficacy of pharmacological therapies for the treatment of opioid-induced constipation: systematic review and meta-analysis. Am J Gastroenterol. 2013; 108(10): 1566-74; quiz 1575, doi: 10.1038/ajg.2013.169, indexed in Pubmed: 23752879.

19. Webster L, Dhar S, Eldon M, et al. A phase 2, double-blind, randomized, placebo-controlled, dose-escalation study to evaluate the efficacy, safety, and tolerability of naloxegol in patients with opioid-induced constipation. Pain. 2013; 154(9): 1542-1550, doi: 10.1016/j. pain.2013.04.024, indexed in Pubmed: 23726675.

20. Chey WD, Webster L, Sostek M, et al. Naloxegol for opioid-induced constipation in patients with noncancer pain. $\mathrm{N}$ Engl J Med. 2014; 370(25): 2387-2396, doi: 10.1056/NEJMoa1310246, indexed in Pubmed: 24896818.

21. Blair HA. Naldemedine: A Review in Opioid-Induced Constipation. Drugs. 2019; 79(11): 1241-1247, doi: 10.1007/s40265019-01160-7, indexed in Pubmed: 31267482.

22. Kanemasa T, Koike K, Arai T, et al. Pharmacologic effects of naldemedine, a peripherally acting $\mu$-opioid receptor antagonist, in in vitro and in vivo models of opioid-induced constipation. Neurogastroenterol Motil. 2019; 31(5): e13563, doi: 10.1111/nmo.13563, indexed in Pubmed: 30821019.

23. Watari R, Matsuda A, Ohnishi S, et al. Minimal contribution of P-gp on the low brain distribution of naldemedine, a peripherally acting $\mu$-opioid receptor antagonist. Drug Metab Pharmacokinet. 2019; 34(2): 126-133, doi: 10.1016/j. dmpk.2018.12.002, indexed in Pubmed: 30770183.

24. Kubota R, Fukumura K, Wajima T. Population Pharmacokinetics and Exposure-Response Relationships of Naldemedine. Pharm Res. 2018; 35(11): 225, doi: 10.1007/s11095018-2501-7, indexed in Pubmed: 30280262.

25. Fukumura K, Yamada T, Yokota T, et al. The Influence of Renal or Hepatic Impairment on the Pharmacokinetics, Safety, and Tolerability of Naldemedine. Clin Pharmacol Drug Dev. 2019 [Epub ahead of print], doi: 10.1002/cpdd.690, indexed in Pubmed: 30977959.

26. Rizmoic. Summary of Product Characteristics. https://www. ema.europa.eu/en/documents/product-information/rizmoic-epar-product-information en.pdf (6.09.2019). 
27. Webster LR, Yamada T, Arjona Ferreira JC. A Phase 2b, Randomized, Double-Blind Placebo-Controlled Study to Evaluate the Efficacy and Safety of Naldemedine for the Treatment of Opioid-Induced Constipation in Patients with Chronic Noncancer Pain. Pain Med. 2017; 18(12): 2350-2360, doi: 10.1093/pm/pnw325, indexed in Pubmed: 28371937.

28. Katakami N, Oda K, Tauchi K, et al. Phase Ilb, Randomized, Double-Blind, Placebo-Controlled Study of Naldemedine for the Treatment of Opioid-Induced Constipation in Patients With Cancer. J Clin Oncol. 2017; 35(17): 1921-1928, doi: 10.1200/JCO.2016.70.8453, indexed in Pubmed: 28445097.

29. Hale M, Wild J, Reddy J, et al. Naldemedine versus placebo for opioid-induced constipation (COMPOSE-1 and COMPOSE-2): two multicentre, phase 3, double-blind, randomised, parallel-group trials. Lancet Gastroenterol Hepatol. 2017; 2(8): 555-564, doi: 10.1016/S2468-1253(17)30105-X, indexed in Pubmed: 28576452.

30. Webster LR, Nalamachu S, Morlion B, et al. Long-term use of naldemedine in the treatment of opioid-induced constipation in patients with chronic noncancer pain: a randomized, double-blind, placebo-controlled phase 3 study. Pain. 2018; 159(5): 987-994, doi: 10.1097/j. pain.0000000000001174, indexed in Pubmed: 29419653.

31. Saito Y, Yokota T, Arai M, et al. Naldemedine in Japanese patients with opioid-induced constipation and chronic noncancer pain: open-label Phase III studies. J Pain Res. 2019; 12: 127-138, doi: 10.2147/JPR.S175900, indexed in Pubmed: 30613161.

32. Wild J, Yamada T, Arjona Ferreira JC, et al. Onset of action of naldemedine in the treatment of opioid-induced constipation in patients with chronic noncancer pain: results from 2 randomized, placebo-controlled, phase 3 trials. Pain. 2019 [Epub ahead of print], doi: 10.1097/j. pain.0000000000001629, indexed in Pubmed: 31145214.

33. Katakami N, Harada T, Murata T, et al. Randomized Phase III and Extension Studies of Naldemedine in Patients With Opioid-Induced Constipation and Cancer. J Clin Oncol. 2017; 35(34): 3859-3866, doi: 10.1200/JCO.2017.73.0853, indexed in Pubmed: 28968171.

34. Katakami N, Harada T, Murata T, et al. Randomized phase 3 and extension studies. Efficacy and impacts on quality of life of naldemedine in subjects with opioid-induced constipation and cancer. Ann Oncol. 2018 [Epub ahead of print], doi: 10.1093/annonc/mdy118, indexed in Pubmed: 29912271.

35. Osaka I, Ishiki H, Yokota T, et al. Safety and efficacy of naldemedine in cancer patients with opioid-induced constipation: a pooled, subgroup analysis of two randomised controlled studies. ESMO Open. 2019; 4(4): e000527, doi: 10.1136/esmoopen-2019-000527, indexed in Pubmed: 31423335.

36. Leppert W. Emerging therapies for patients with symptoms of opioid-induced bowel dysfunction. Drug Des Devel Ther. 2015; 9: 2215-2231, doi: 10.2147/DDDT.S32684, indexed in Pubmed: 25931815.

37. Leppert $\mathrm{W}$, Woron J. The role of naloxegol in the management of opioid-induced bowel dysfunction. Therap Adv Gastroenterol. 2016; 9(5): 736-746, doi: 10.1177/1756283X16648869, indexed in Pubmed: 27582887.

38. Stern EK, Brenner DM. Spotlight on naldemedine in the treatment of opioid-induced constipation in adult patients with chronic noncancer pain: design, development, and place in therapy. J Pain Res. 2018; 11: 195-199, doi: 10.2147/JPR.S141322, indexed in Pubmed: 29391826.

39. Dzierżanowski T. The role of peripherally acting mu-opioid receptor antagonists (PAMORA) in the treatment of constipation in palliative care. Palliative Medicine. 2019; 11(2): 51-57, doi: 10.5114/pm.2019.86528.

40. Larkin PJ, Cherny NI, La Carpia D, et al. ESMO Guidelines Committee. Diagnosis, assessment and management of constipation in advanced cancer: ESMO Clinical Practice Guidelines. Ann Oncol. 2018; 29(Suppl 4): iv111-iv125, doi: 10.1093/annonc/mdy148, indexed in Pubmed: 30016389.

41. Leppert W, Dzierżanowski T, Stachowiak A, et al. Constipation in cancer patients - the management recommendations of the Expert Group of the Polish Association for Palliative Medicine. Med Paliat. 2014; 6: 1-10.

42. Luthra P, Burr NE, Brenner DM, et al. Efficacy of pharmacological therapies for the treatment of opioid-induced constipation: systematic review and network meta-analysis. Gut. 2018 [Epub ahead of print], doi: 10.1136/gutjnl-2018-316001, indexed in Pubmed: 29730600.

43. Esmadi M, Ahmad D, Hewlett A. Efficacy of naldemedine for the treatment of opioid-induced constipation: A meta-analysis. J Gastrointestin Liver Dis. 2019; 28(1): 41-46, doi: 10.15403/jgld.2014.1121.281.any, indexed in Pubmed: 30851171. 